\title{
Epidemiology, Occupation
}

National Cancer Institute

\section{Source}

National Cancer Institute. Epidemiology, Occupation. NCI Thesaurus. Code C18771.

The consideration of occupation as a variable in disease incident, transmission, and control. 